\title{
SELF-CONSISTENT AVERAGE DENSITY MATRICES AND THE STRUTINSKY ENERGY THEOREM
}

\author{
M. BRACK and P. QUENTIN* \\ The Niels Bohr Institute, Copenhagen, Denmark
}

Received 19 March 1975

\begin{abstract}
For nuclei ranging from ${ }^{16} \mathrm{O}$ to ${ }^{208} \mathrm{~Pb}$ and for the Skyrme III effective interaction, it is shown that the sum of the average energy plus the first order shell correction reproduces perfectly well the exact Hartree-Fock energy, if the average density matrices are calculated self-consistently.
\end{abstract}

In recent extended calculations $[1,2]$ we have investigated numerically the validity of the Strutinsky shell-correction method [3] within the framework of the Hartree-Fock (HF) approximation. We followed there the program outlined by Strutinsky [3] in deriving the shell-correction expansion of the total HF energy:

$E_{\mathrm{HF}}(\rho)=\bar{E}(\rho)+\delta E_{1}+\delta E_{2}$.

Here $\bar{E}(\bar{\rho})$ is the average ("liquid drop") energy defined by the smooth part $\bar{\rho}$ of the self-consistent density matrix $\rho ; \delta E_{1}$ is the first order shell-correction found in the usual way from the eigenvalues $\hat{\epsilon}_{i}$ of the averaged one body HF-Hamiltonian $\bar{H}(\bar{\rho})$; and $\delta E_{2}$ collects all terms of second and higher orders in the fluctuating part $\delta \rho=\rho-\bar{\rho}$ of the density matrix. The term $\delta E_{2}$ is neglected in the usual shell-correction approach.

In the calculations of refs. [1, 2], performed with various effective interactions for many different nuclei, it was indeed found that $\delta E_{2}$ plays a relatively unimportant role for medium and heavy nuclei. There the quantity $\delta E_{2}$, as a function of both deformation and nucleon numbers, fluctuates not more than by $\pm \sim 1 \mathrm{MeV}$ around a constant value of $\sim 1.5 \mathrm{MeV}$. Furthermore, the energy $\bar{E}(\bar{\rho})$ was found to have all the typical features of a liquid drop model energy.

In this letter we want to present an alternative way of obtaining an expression of the form (1). As discussed in ref. [2], one can define the quantities $\bar{E}$ and $\delta E_{1}$ in slightly different ways which alter them

\footnotetext{
* Permanent address: Division de Physique théorique, I.P.N., Orsay, France.
}

only by terms of second and higher orders in $\delta \rho$. We choose here to introduce a self-consistent average density matrix $\widetilde{\rho}$ which leads to a self-consistent smooth part of the total energy. We define $\tilde{\rho}$ by replacing the HF occupation numbers in the exact one body density matrix $\rho$ by the average occupation numbers $\widetilde{n}_{i}$ used in the Strutinsky energy smoothing procedure (see e.g. ref. [4]). This can indeed be done iteratively following the general variational method outlined by Vautherin [5]. In fact, such a procedure resembles formally to the variational inclusion of some pairing correlations, as currently practised $[1,2$, $5,6]$, or to the one used in HF calculations at finite temperatures [7]. The occupation numbers $\widetilde{n_{i}}$ differ, however, from the pairing or temperature occupation probabilities in one important aspect: due to the inclusion of the curvature-corrections and the fulfilment of the plateau (or stationary) condition [4], the Strutinsky smoothing leads to a cold average, i.e. no excitation energy is brought into the nucleus by it (see e.g. ref. [8] for a discussion of this point).

The possibility of introducing a self-consistent semiclassical energy in connection with the Strutinsky method has also been considered theoretically by Tyapin [9]. In the droplet model calculations of Myeres [10], the self-consistency problem was solved within the Thomas-Fermi approximation. The Strutinsky energy averaging has been shown to be equivalent to an extended Thomas-Fermi approximation [11], thus including semiclassical corrections to the pure Thomas-Fermi model which are necessary to describe correctly the average surface properties of finite nuclei.

After solving the variational equations one finds 
Table 1

Zero, first and higher order terms in the Strutinsky energy expansion for five nuclei calculated at their ground states with the Skyrme III force. All energies are expressed in MeV. The energy reference is the HF energy $E_{\text {HF }}$ listed in column 2 . In columns 3,5 and 7 are reported the quantities corresponding to the expansion (3) of $E_{H F}$, whereas those associated with the usual expansion (1) are to be found in columns 4,6 and 8. Pairing correlations are included using the uniform gap method [3]. The basis of expansion of the single particle HF states corresponds to 7 oscillator shells for the nuclei ${ }^{16} \mathrm{O}$ and ${ }^{40} \mathrm{Ca}, 9$ for the nucleus ${ }^{90} \mathrm{Zr}, 11$ for the nuclei ${ }^{168} \mathrm{Yb}$ and ${ }^{208} \mathrm{~Pb}$.

\begin{tabular}{cccccccc}
\hline Nucleus & $E_{\mathrm{HF}}$ & $\tilde{E}$ & $\bar{E}$ & $\delta E_{1}\left(\tilde{\epsilon}_{i}\right)$ & $\delta E_{1}\left(\hat{\epsilon}_{i}\right)$ & $\delta E_{2}(\tilde{\rho})$ & $\delta E_{2}(\bar{\rho})$ \\
\hline${ }^{16} \mathrm{O}$ & -126.8 & -122.1 & -122.7 & -4.7 & -5.7 & 0.0 & 1.6 \\
${ }^{40} \mathrm{Ca}$ & -339.6 & -337.5 & -338.1 & -2.7 & -4.8 & 0.5 & 3.3 \\
${ }^{90} \mathrm{Zr}$ & -779.2 & -774.3 & -775.7 & -5.5 & -5.8 & 0.6 & 2.3 \\
${ }^{168} \mathrm{Yb}$ & -1352.2 & -1349.6 & -1349.9 & -3.0 & -4.3 & 0.3 & 2.0 \\
${ }^{208} \mathrm{~Pb}$ & -1625.4 & -1606.6 & -1607.1 & -19.2 & -20.6 & 0.5 & 2.3 \\
\hline
\end{tabular}

the average density matrix $\tilde{\rho}$ and the self-consistent average energy

$\widetilde{E}=E_{\mathrm{HF}}(\tilde{\rho})=\operatorname{tr} \tau \tilde{\rho}+\frac{1}{2} \operatorname{tr} \operatorname{tr} \tilde{\rho} \mathcal{\nu} \tilde{\rho}$

where $\tau$ is the matrix of the kinetic energy operator and $\mathcal{V}$ the antisymmetrized matrix of the effective nucleon interaction (which here for simplicity is assumed to be a two body interaction). We will denote the eigenvalues of the corresponding variational one body Hamiltonian $\tilde{H}(\tilde{\rho})$ by $\tilde{\epsilon}_{i}$. Starting from the exact (i.e. not averaged) $\mathrm{HF}$ energy $E_{\mathrm{HF}}(\rho)$, one can rewrite eq. (1) as

$E_{\mathrm{HF}}=E_{\mathrm{HF}}(\rho)=E_{\mathrm{HF}}(\tilde{\rho})+\delta E_{1}\left(\tilde{\epsilon}_{i}\right)+\delta E_{2}(\tilde{\rho})$

where

$$
\delta E_{1}\left(\widetilde{\epsilon}_{i}\right)=\sum_{i=1}^{N} \widetilde{\epsilon}_{i}-\sum_{i} \widetilde{\epsilon}_{i} \tilde{n}_{i}
$$

is the usual shell correction and $\delta E_{2}(\tilde{\rho})$ collects again all higher order terms. Eq. (3) assumes of course that the averaged and the exact HF solutions have been constrained to the same deformation.

In our numerical calculations we have used the effective interaction of Skyrme in the version S III [12]. Pairing correlations are included self-consistently in the exact $\mathrm{HF}$ energy $E_{\mathrm{HF}}$, in the way proposed by Vautherin [5]. The constant pairing matrix elements $G_{\mathrm{p}}$ and $G_{\mathrm{n}}$ are determined using the average gap $\tilde{\Delta}$ as a constant parameter [3]. A pairing correction is also added to the shell-correction $\delta \mathrm{E}_{1}\left(\tilde{\epsilon}_{i}\right)$ in the usual way [3].Technical details of the constrained HF calculations as well as the modifications of eqs. (3) and

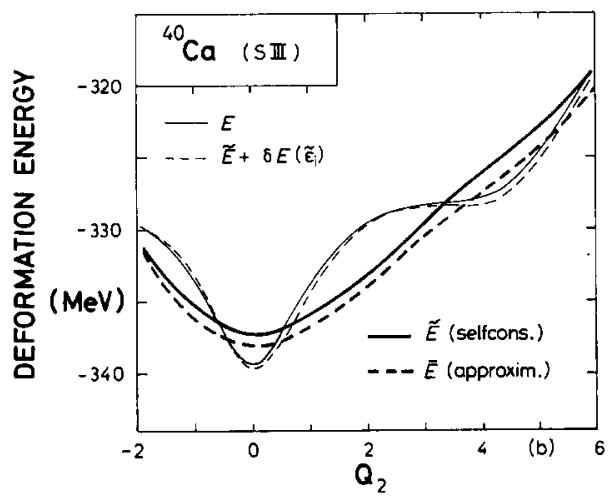

Fig. 1. Deformation energy curve for the ${ }^{40} \mathrm{Ca}$ nucleus, obtained with the Skyrme III force. Some pairing correlations have been included $(\widetilde{\Delta}=1 \mathrm{MeV})$. The basis of expansion of the single particle HF states corresponds for the spherical solution to 7 oscillator shells. The thin solid line is the HF energy $E_{\mathrm{HF}}$, and the heavy dashed line the average energy $\bar{E}$ defined in (1). The heavy solid line is the self-consistent average energy $\widetilde{E}$ defined in (2) and the thin dashed line is the approximation $\widetilde{E}+\delta E_{1}\left(\widetilde{\epsilon}_{i}\right)$ to the HF energy.

(4) due to the pairing correlations and the density dependence of the interaction can be found in refs. [6] and [1].

The results for the average energy $\widetilde{E}$ are rather similar to the earlier results for $\bar{E}$ obtained when using the non-self-consistent average density matrix $\bar{\rho}$. The self-consistency of $\widetilde{\rho}$ has, generally, a more important influence on the shell-corrections: the first-order correction $\delta \mathrm{E}_{1}\left(\tilde{\epsilon}_{i}\right)$ is found to contain essentially all fluctuations of the total energy $E_{\mathrm{HF}}$, the remaining term $\delta \mathrm{E}_{2}(\tilde{\rho})$ being very small and nearly constant. 


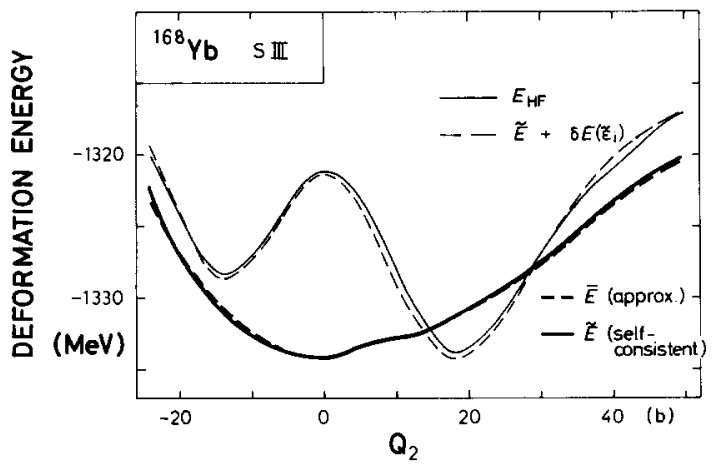

Fig. 2. The same as in fig. 1 , but for the ${ }^{168} \mathrm{Yb}$ nucleus.

In table 1 , the HF energies and the single terms of the two expansions (1), (3) are lsietdd for 5 nuclei ranging from ${ }^{16} \mathrm{O}$ to ${ }^{208} \mathrm{~Pb}$ in their ground states. It is seen that the values of $\delta E_{2}(\tilde{\rho})$ vary only from $\sim 0.0$ to $0.6 \mathrm{MeV}$, thus containing almost no oscillations, whereas the values $\delta E_{2}(\bar{\rho})$ vary more.

This effect is even more striking when the deformation dependence is considered. Figs. 1 and 2 show deformation energy curves obtained with a quadratic constraint on the mass quadrupole moment $Q_{2}$ for the nuclei ${ }^{40} \mathrm{Ca}$ and ${ }^{168} \mathrm{Yb}$. Both the exact and the averaged self-consistent energies, $E_{\mathrm{HF}}$ and $\widetilde{E}$, are shown. The non-self-consistent average energies $\bar{E}$, obtained as in refs. $[1,2]$, are also plotted. The curves $\widetilde{E}+\delta E_{1}\left(\widetilde{\epsilon}_{i}\right)$ approximate the exact $\mathrm{HF}$ energy curve $E_{\mathrm{HF}}$ very closely at all points, thus showing the smallness of the term $\delta E_{2}(\tilde{\rho})$. Such a result is particularly spectacular for the light nucleus ${ }^{40} \mathrm{Ca}$. For this nucleus indeed, in the usual expansion (1), $\delta E_{2}$ was found [2] to be of the same order as $\delta E_{1}$, namely oscillating between 0 and $3 \mathrm{MeV}$.

We have here presented some numerical evidence that one can define the average density matrix in such a way that the Strutinsky energy expansion (3) can be truncated already after the first order term in a very good approximation. Furthermore with the selfconsistent definition of the bulk part of $\rho$, the domain of validity of the Strutinsky method has been extended to nuclei as light as ${ }^{40} \mathrm{Ca}$ or ${ }^{16} \mathrm{O}$.

It may also be stressed that we have provided here a completely consistent calculation of the semiclassical solution associated with a given microscopic Hamiltonian. The way it has been obtained here is far from being cheap, since it needs as much computational time as complete microscopic calculation. However, the results presented above could have interesting practical consequences. First of all, one could perform usual Strutinsky calculations using the smoothed single particle Hamiltonian $\widetilde{H}(\tilde{\rho})$ and the corresponding liquid drop energy $\widetilde{E}$ as inexpensive substitutes of HF calculations in the vicinity of some selected nuclei (where complete HF calculations would be achieved). Secondly, if one is able to realize the self-consistent variation only for the semiclassical part of the solution (e.g. in the extended Thomas-Fermi approximation of ref. [11]) one would be able to produce precise estimates of the total HF energy by simply adding a standard shell-correction calculation.

We would like to thank Professor R.K. Bhaduri and Doctor B.K. Jennings for interesting discussions. The hospitality extended to us at the Niels Bohn Institute, together with the support from the Japan World Exposition Commemorative Fund is gratefully acknowledged.

\section{References}

[1] M. Brack and P. Quentin, Physics and chemistry of fission 1973, Rochester (IAEA, Vienna, 1974) Vol. I, p. 231.

[2] M. Brack and P. Quentin, Conf. on Hartree-Fock and self-consistent field theories in nuclei, Trieste, 1975.

[3] V.M. Strutinsky, Nucl. Phys. A95 (1967) 420; A122 (1968) 1.

[4] M. Brack and H.C. Pauli, Nucl. Phys. A207 (1973) 401.

[5] D. Vautherin, Phys. Rev. C7 (1973) 296.

[6] H. Flocard, P. Quentin, A.K. Kerman and D. Vautherin, Nucl. Phys. A203 (1973) 433.

[7] M. Brack and P. Quentin, Phys. Lett. 52B (1974) 159; Physica Scripta 10A, in print.

[8] M. Brack, Proc. Intern. Summer School in Nuclear 'Physics, Predeal, Romania, 1974.

[9] A.S. Tyapin, Yad. Fiz. 19 (1974) 263 (Sov. J. Nucl. Phys. 19 (1974) 129).

[10] W.D. Myers, Nucl. Phys. A145 (1969) 387; W.D. Myers and W.J. Swiatecki, Ann. Phys. 55 (1969) 395.

[11] B.K. Jennings, Nucl. Phys. A207 (1973) 401; B.K. Jennings and R.K. Bhaduri, Nucl. Phys. A237 (1975) 149.

[12] M. Beiner, H. Flocard, Nguyen van Giai and P. Quentin, Nucl. Phys. A238 (1975) 433. 